\title{
Three-Dimensional FDTD Modeling of Impulsive ELF Propagation About the Earth-Sphere
}

\author{
Jamesina J. Simpson, Student Member, IEEE, and Allen Taflove, Fellow, IEEE
}

\begin{abstract}
This paper reports the application of an efficient finite-difference time-domain (FDTD) algorithm to model impulsive extremely low frequency (ELF) propagation within the entire Earth-ionosphere cavity. Periodic boundary conditions are used in conjunction with a three-dimensional latitude-longitude FDTD space lattice which wraps around the complete Earth-sphere. Adaptive combination of adjacent grid cells in the east-west direction minimizes cell eccentricity upon approaching the poles and hence maintains Courant stability for relatively large time steps. This technique permits a direct, three-dimensional time-domain calculation of impulsive, round-the-world ELF propagation accounting for arbitrary horizontal as well as vertical geometrical and electrical inhomogeneities/anisotropies of the excitation, ionosphere, lithosphere, and oceans. The numerical model is verified by comparing its results for ELF propagation attenuation with corresponding data reported in the literature.
\end{abstract}

Index Terms-Earth, extremely low frequency (ELF), finite-difference time-domain (FDTD) methods, propagation, sphere.

\section{INTRODUCTION}

$\mathbf{P}$ ROPAGATION of extremely low-frequency (ELF: $3 \mathrm{~Hz}-3$ $\mathrm{kHz}$ ) and very low-frequency (VLF: $3-30 \mathrm{kHz}$ ) electromagnetic waves in the Earth-ionosphere waveguide is a problem having a rich history of theoretical investigation extending over many years (see, for example, [1]-[6]). Currently, ELF/VLF propagation phenomena form the physics basis of important remote-sensing investigations of lightning and sprites [7], global temperature change [8], subsurface structures [9], and potential earthquake precursors [10].

Most theoretical techniques for modeling ELF/VLF propagation in the Earth-ionosphere waveguide are based upon frequency-domain waveguide mode theory [2], [5]. Berenger [11], [12] and Cummer [13] were the first investigators to apply the finite-difference time-domain (FDTD) method [14] to this problem. They used two-dimensional (2-D) cylindrical-coordinate FDTD grids to investigate VLF propagation over lossy ground paths due to either manmade sources or lightning discharges. Both investigators reported very good agreement of their FDTD models with benchmark data. In particular, Cummer found "extremely good" agreement between numerical mode theory and FDTD for nighttime ELF-VLF spectra below $10 \mathrm{kHz}$, and a comparable level of agreement for the

Manuscript received November 26, 2002; revised March 30, 2003. This work was supported by the Pittsburgh Supercomputing Center under Grant ECS0200006P.

The authors are with the Department of Electrical and Computer Engineering, Northwestern University, Evanston, IL 60208 USA (e-mail: j-simpson @ northwestern.edu)

Digital Object Identifier 10.1109/TAP.2004.823953 daytime spectra. A "major strength" of FDTD is its automatic calculation of all the fields due to lightning (discharge and postdischarge, evanescent and propagating), while other solution techniques are forced to treat these fields separately. Further, FDTD can, in principle, permit straightforward modeling (with no increase in simulation time) of arbitrary horizontal as well as vertical inhomogeneities of the atmosphere and Earth. Cummer concluded that "the simplicity of FDTD propagation modeling and ever-increasing computer power probably make FDTD the technique of the future."

Subsequently, Simpson and Taflove [15]-[17] ${ }^{1}$ and Hayakwa et al. [18], [19] reported the initial applications of FDTD to model the complete Earth-sphere at ELF. Both groups used spherical-coordinate latitude-longitude grids with periodic boundary conditions based upon fundamental work by Holland [20]. Hayakawa et al. reported no improvements relative to Holland's grid, which is subject to increasing space-cell eccentricity upon approaching the poles due to converging lines of longitude. Since the Courant stability limit is set by the smallest space-cell dimension in a grid, this eccentricity mandates a corresponding reduction in the allowable time step. For the Holland grid, the Courant limit is reduced to approximately $d / r_{e}$ times the limit that would exist if all grid cells maintained the square configuration of those located at the equator, where $d$ is the cell dimension at the equator and $r_{e}$ is the radius of the Earth. This is a very significant mandatory time-step reduction factor for high-resolution grids having $d$ smaller than $100 \mathrm{~km}$ (i.e., $d / r_{e}<1 / 64$ ).

In contrast, in [15] and [16], Simpson and Taflove reported a key improvement in FDTD mesh generation to minimize the effects of increasing space-cell eccentricity upon approaching a pole. Their mesh was constructed to maintain approximately square cells in the polar regions by a novel adaptive cell-combining technique applied to adjacent grid-cells in the east-west direction. This permitted maintenance of the time-step at nearly the level permitted by the Courant stability condition for the square equatorial cells. Further, as shown in [15] and [16], their technique demonstrated a high degree of isotropy for numerical wave propagation within the model despite the mesh nonuniformity due to the adaptive east-west cell combinations. This technique, the basis of the work reported in this paper, permits a standard laboratory workstation (the Dell 530) to generate high-resolution (approximately $40 \times 40 \times 5 \mathrm{~km}$ ) modeling results for global, fully three-dimensional (3-D) impulsive ELF propagation within the entirety of the Earth-ionosphere cavity.

This paper reports for the first time the complete algorithmic details of the extension of the work of [15] and [16] to a fully 3-D

\footnotetext{
${ }^{1}$ http://www.ece.northwestern.edu/ecefaculty/taflove/3Dmovietext@gif.avi.
} 


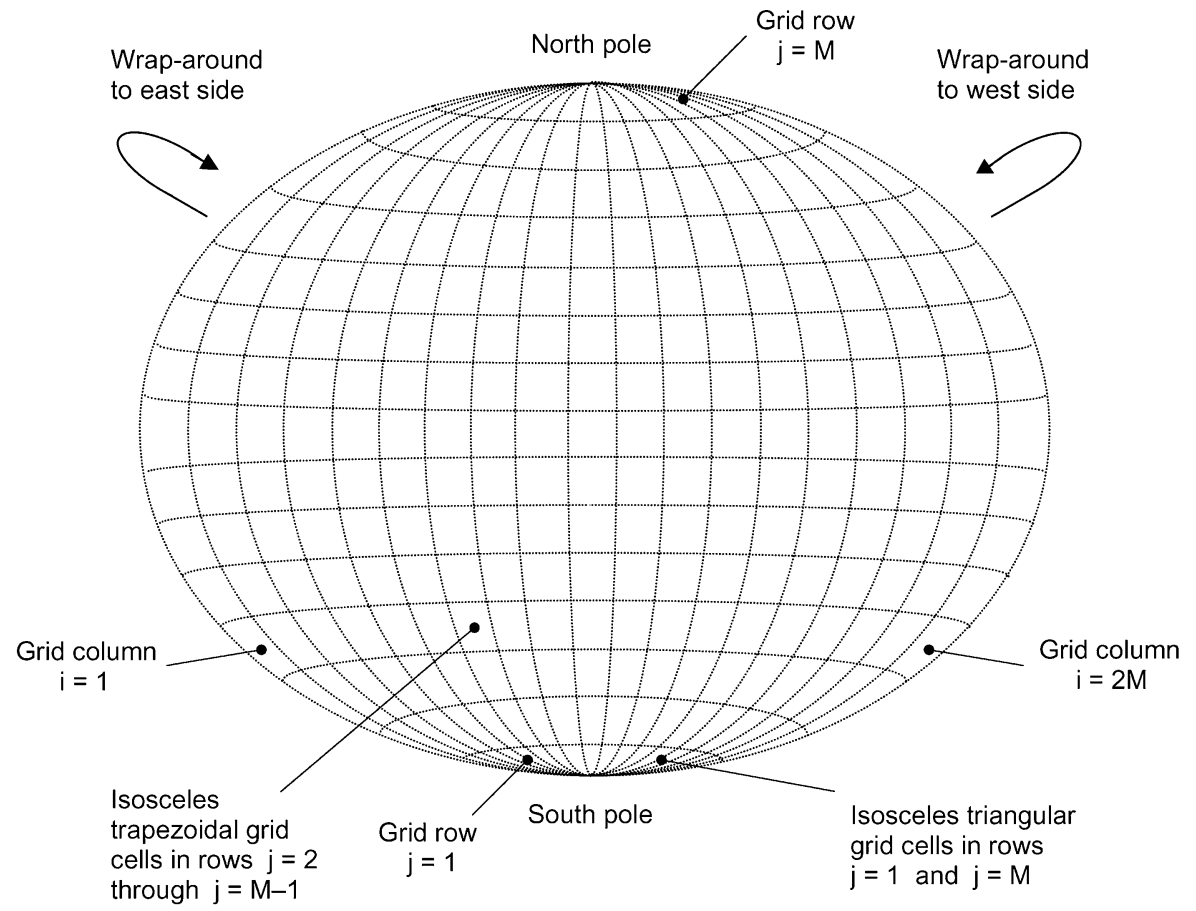

Fig. 1. General layout of the of the three-dimensional FDTD lattice covering the complete Earth-sphere as seen in a TM plane at a constant radial coordinate.

model of the complete Earth-ionosphere cavity. To our knowledge, this represents the first direct time-domain model for ELF around-the-world propagation with sufficiently fine grid resolution (approximately $40 \times 40 \times 5 \mathrm{~km}$ ) to render fine-grained local details of the complex horizontal and vertical geometrical and electrical inhomogeneities/anisotropies of the ionosphere, lithosphere, and oceans.

Section II introduces the 3-D FDTD space lattice used in this model. Sections III and IV discuss the details of the corresponding numerical algorithm. Section $\mathrm{V}$ reports a validation study that compares the FDTD calculations for frequency-dependent ELF propagation attenuation with data reported in the literature. This section provides details of the impulsive excitation and the assumed Earth-ionosphere geometry and electrical properties. Section VI concludes with a discussion of ongoing and potential research in this area.

\section{FDTD SPACE LATTICE}

The present model maps the complete Earth-ionosphere cavity onto a 3-D spherical-coordinate FDTD space lattice that extends $\pm 100 \mathrm{~km}$ radially from sea level. Fig. 1 illustrates the general layout of the lattice as seen from the TM plane at a constant radial coordinate. The lattice is a logically Cartesian $2 M \times M \times K$ cell arrangement, where $M$ is a power of two. The lattice-cell position index in the west-to-east direction is $1 \leq i \leq 2 M$, the lattice-cell position index in the south-to-north direction is $1 \leq j \leq M$, and the lattice-cell position index in the radial direction is $1 \leq k \leq K$. We see that the grid cells follow along lines of constant latitude, $\theta=$ constant, where $\theta$ is the usual spherical angle measured from the north pole; and along lines of constant longitude, $\phi=$ constant, where $\phi$ is the usual spherical azimuthal angle measured from a specified prime meridian. In this manner, each TM plane of the grid shown in Fig. 1 is comprised of isosceles trapezoidal cells away from the north and south poles [Fig. 2(a)] and isosceles triangular cells at the poles [Fig. 2(b)]. Similarly, each transverse electric (TE) plane at a constant radial coordinate is comprised of isosceles trapezoidal cells away from the north and south poles [Fig. 3(a)] and a polygon at the poles [Fig. 3(b)].

We choose to have the same angular increment in latitude, $\Delta \theta=\pi / m$, for each cell in the grid. Thus, the south-north span of each trapezoidal or triangular grid cell is $\Delta_{\mathrm{s}-\mathrm{n}}=\pi R / \mathrm{m}$, where $R$ is the radial distance from the center of the Earth. To maintain square or nearly square grid cells near the equator, we select the baseline value of the angular increment in longitude $\Delta \phi$ to equal $\Delta \theta$. However, this causes the west-east span of each cell $\Delta_{\mathrm{w}-\mathrm{e}}=R \Delta \phi \sin \theta$ to be a function of $\theta$. This could be troublesome for cells near the north and south poles where $\theta \rightarrow 0$ and $\theta \rightarrow \pi$, respectively. There, the geometrical eccentricity of each cell $\Delta_{\mathrm{s}-\mathrm{n}} / \Delta_{\mathrm{w}-\mathrm{e}}=\Delta \theta /(\Delta \phi \sin \theta)$ would become quite large, and the numerical stability and efficiency of the FDTD algorithm would be degraded. An algorithmic means to deal with this problem will be discussed below.

The wraparound or joining of the lattice is along a specific line of constant longitude, or meridian. As discussed below, this joining is, in effect, a periodic boundary condition applied at each $j$-row of lattice cells, whether trapezoids or triangles.

\section{FDTD Algorithm, $\mathrm{TM}_{\mathrm{z}}$ COMPONENTS}

\section{A. Basic Algorithm}

Given the above assumptions, Ampere's law in integral form [14] can be applied to develop an FDTD time-stepping relation 


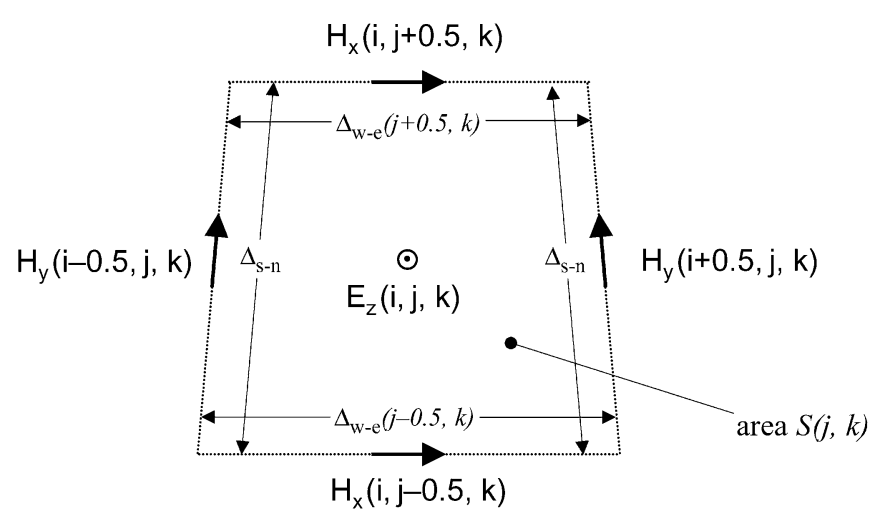

(a)

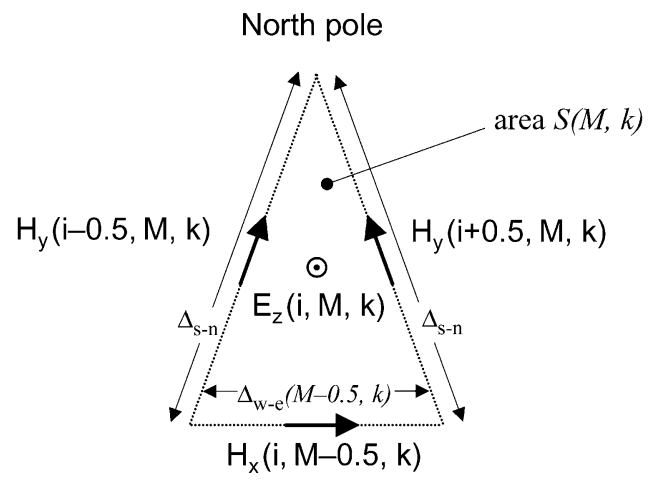

(b)

Fig. 2. Details of the $\mathrm{TM}_{\mathrm{z}}$-plane lattice-cell geometry: (a) isosceles trapezoidal cell in the northern hemisphere away from the north pole and (b) isosceles triangular cell at the north pole.

for the electric field $E_{z}$ at the center of the $(i, j, k)$ th trapezoidal grid cell. For example, referring to Fig. 2(a), we have

$$
\begin{aligned}
E_{z}^{n+1}(i, j, k)=E_{z}^{n} & (i, j, k)+\frac{\Delta t}{\varepsilon_{0} S(j, k)} \\
\times & \left\{H_{x}^{n+0.5}(i, j-0.5, k) \Delta_{\mathrm{w}-\mathrm{e}}(j-0.5, k)\right. \\
& -H_{x}^{n+0.5}(i, j+0.5, k) \Delta_{\mathrm{w}-\mathrm{e}}(j+0.5, k) \\
& +\left[H_{y}^{n+0.5}(i+0.5, j, k)\right. \\
& \left.\left.-H_{y}^{n+0.5}(i-0.5, j, k)\right] \Delta_{\mathrm{s}-\mathrm{n}}\right\}
\end{aligned}
$$

where $\Delta t$ is the time-step and

$$
\begin{aligned}
\Delta_{\mathrm{w}-\mathrm{e}}(j+0.5, k)= & R \Delta \phi \sin \left[(M-j) \frac{\pi}{M}\right] \\
\Delta_{\mathrm{w}-\mathrm{e}}(j-0.5, k)= & R \Delta \phi \sin \left[(M-j+1) \frac{\pi}{M}\right] \\
S(j, k)= & {\left[\Delta_{\mathrm{w}-\mathrm{e}}(j-0.5, k)\right.} \\
& \left.+\Delta_{\mathrm{w}-\mathrm{e}}(j+0.5, k)\right] \frac{\Delta_{\mathrm{s}-\mathrm{n}}}{2} .
\end{aligned}
$$

Similarly, referring to Fig. 2(b), the update for $E_{z}$ at the center of the $i$ th triangular grid cell at the north pole $(j=M)$ is given by

$$
\begin{aligned}
E_{z}^{n+1}(i, M, k)= & E_{z}^{n}(i, M, k)+\frac{\Delta t}{\varepsilon_{0} S(M, k)} \\
\times & \left\{H_{x}^{n+0.5}(i, M-0.5, k)\right. \\
& \times \Delta_{\mathrm{w}-\mathrm{e}}(M-0.5, k) \\
& +\left[H_{y}^{n+0.5}(i+0.5, M, k)\right. \\
& \left.\left.-H_{y}^{n+0.5}(i-0.5, M, k)\right] \Delta_{\mathrm{s}-\mathrm{n}}\right\}
\end{aligned}
$$

where $\Delta_{\mathrm{w}-\mathrm{e}}(M-0.5, k)$ is given by (2b) for the case $j=M$ and

$$
\begin{aligned}
S(M, k)= & \frac{\Delta_{\mathrm{w}-\mathrm{e}}(M-0.5, k) \Delta_{\mathrm{s}-\mathrm{n}}}{2} \\
& \times \sin \left\{\cos ^{-1}\left[\frac{\Delta_{\mathrm{w}-\mathrm{e}}(M-0.5, k)}{2 \Delta_{\mathrm{s}-\mathrm{n}}}\right]\right\} .
\end{aligned}
$$

Expressions analogous to (3) and (4) can be derived for the $i$ th triangular grid cell at the south pole $(j=1)$.

The basic FDTD time-stepping algorithm is completed by specifying the updates for the $H_{x}$ and $H_{y}$ fields. For example, referring to the trapezoidal grid cell shown in Fig. 2(a), we have

$$
\begin{aligned}
H_{x}^{n+1.5}(i, j-0.5, k)= & H_{x}^{n+0.5}(i, j-0.5, k) \\
& +\frac{\Delta t}{\mu_{0} \Delta_{\mathrm{r}}}\left[E_{y}^{n+1}(i, j-0.5, k+0.5)\right. \\
& \left.-E_{y}^{n+1}(i, j-0.5, k-0.5)\right] \\
& +\frac{\Delta t}{\mu_{0} \Delta_{\mathrm{s}-\mathrm{n}}}\left[E_{z}^{n+1}(i, j-1, k)\right. \\
H_{y}^{n+1.5}(i+0.5, j, k)= & H_{y}^{n+0.5}(i+0.5, j, k) \\
& +\frac{\Delta t}{\mu_{0} \Delta_{\mathrm{r}}}\left[E_{x}^{n+1}(i+0.5, j, k-0.5)\right. \\
& \frac{\left.-E_{x}^{n+1}(i+0.5, j, k+0.5)\right]}{\mu_{0} \Delta_{\mathrm{w}-\mathrm{e}}(j, k)}\left[E_{z}^{n+1}(i+1, j, k)\right. \\
& \left.-E_{z}^{n+1}(i, j, k)\right] \quad(6)
\end{aligned}
$$

where $\Delta_{\mathrm{r}}$ is the cell span in the radial direction.

For a triangular grid cell at the north pole $(j=M)$ shown in Fig. 2(b), we similarly have

$$
\begin{aligned}
H_{x}^{n+1.5}(i, M-0.5, k)= & H_{x}^{n+0.5}(i, M-0.5, k) \\
& +\frac{\Delta t}{\mu_{0} \Delta_{\mathrm{r}}}\left[E_{y}^{n+1}(i, M-0.5, k+0.5)\right. \\
& \left.-E_{y}^{n+1}(i, M-0.5, k-0.5)\right] \\
& +\frac{\Delta t}{\mu_{0} \Delta_{\mathrm{s}-\mathrm{n}}}\left[E_{z}^{n+1}(i, M-1, k)\right. \\
H_{y}^{n+1.5}(i+0.5, M, k)= & H_{y}^{n+0.5}(i+0.5, M, k) \\
& +\frac{\Delta t}{\mu_{0} \Delta_{\mathrm{r}}}\left[E_{x}^{n+1}(i+0.5, M, k-0.5)\right. \\
& +\frac{\Delta t}{\mu_{0} \Delta_{\mathrm{w}-\mathrm{e}}(M, k)} \\
& \times\left[E_{z}^{n+1}(i+1, M, k)\right. \\
& \left.-E_{z}^{n+1}(i, M, k)\right] .
\end{aligned}
$$

Expressions analogous to (7) and (8) can be derived for a triangular grid cell at the south pole $(j=1)$.

\section{B. Merging Trapezoidal Grid Cells Approaching the Polar Regions}

As stated earlier, near the north and south poles the geometrical eccentricity of each trapezoidal cell $\Delta_{\mathrm{s}-\mathrm{n}} / \Delta_{\mathrm{w}-\mathrm{e}}=$ $\Delta \theta /(\Delta \phi \sin \theta)$ can become quite large for a constant value of $\Delta \phi$, thereby degrading the numerical stability and efficiency of 


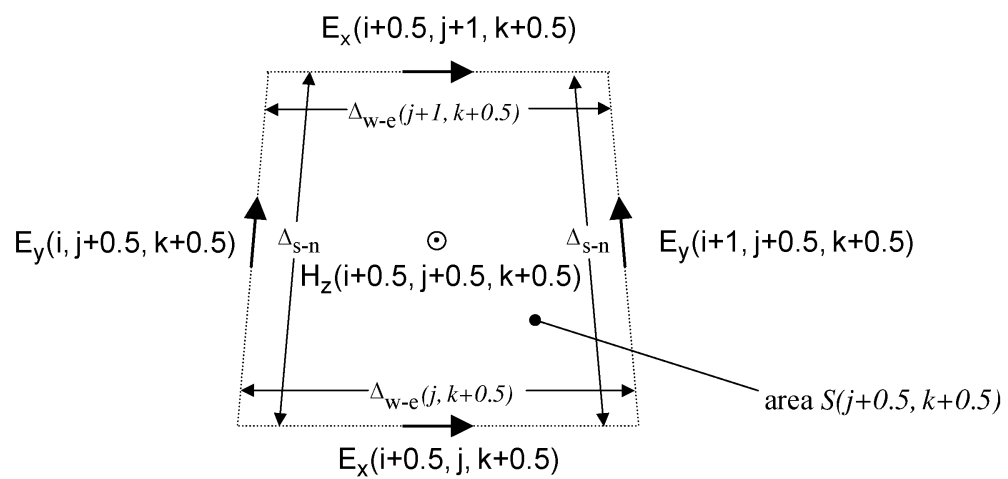

(a)

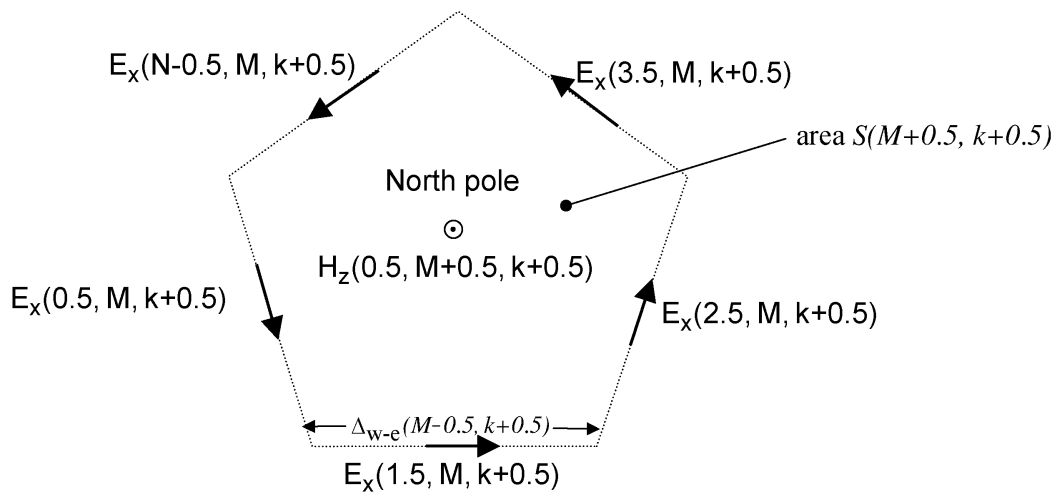

(b)

Fig. 3. Details of the $\mathrm{TE}_{\mathrm{z}}$-plane lattice-cell geometry: (a) isosceles trapezoidal cell in the northern hemisphere away from the north pole and (b) polygon cell directly at the north pole.

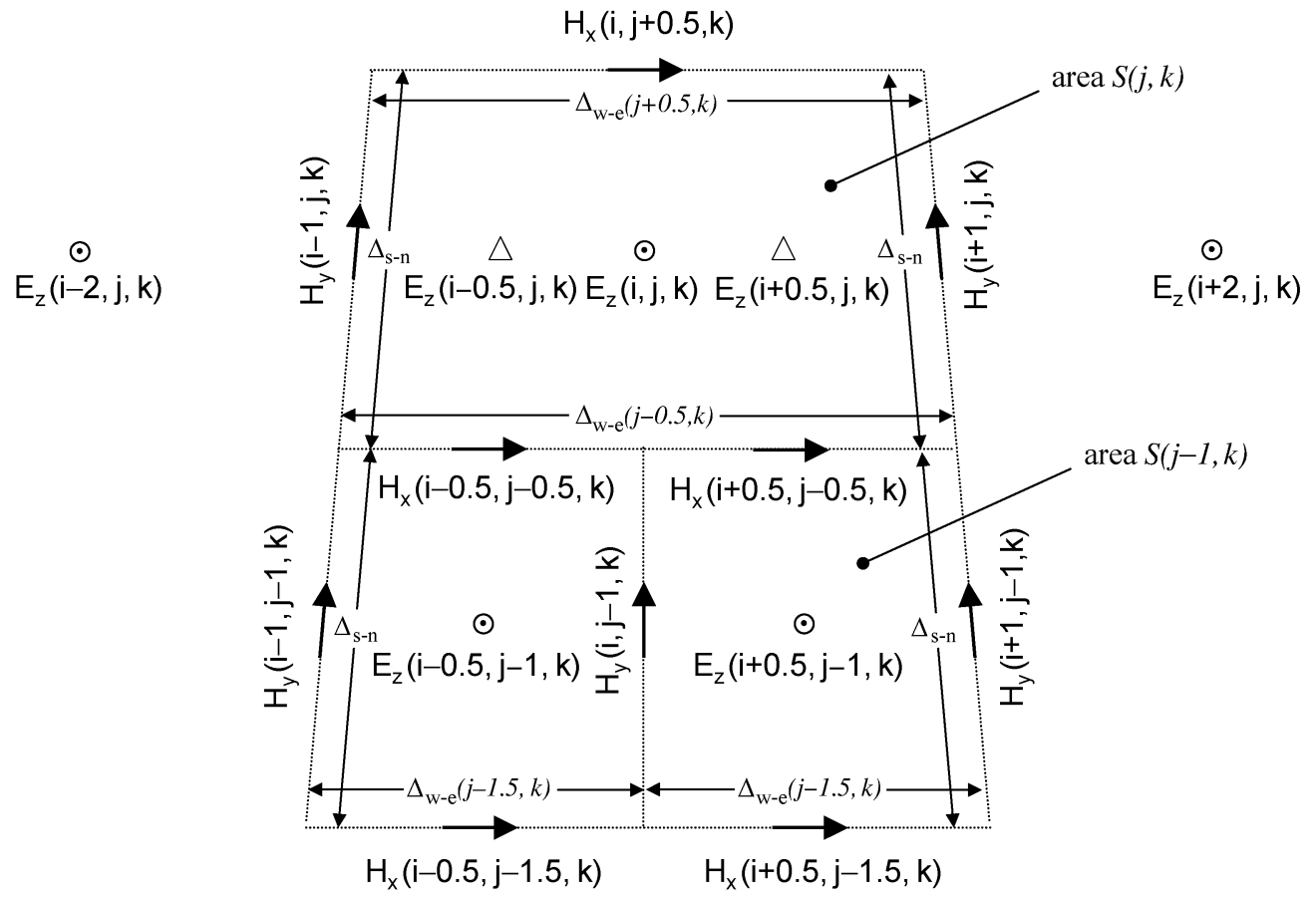

Fig. 4. Details of the $\mathrm{TM}_{\mathrm{z}}$-plane grid-cell geometry in the northern hemisphere at the transition between two adjacent regular cells and a single cell spanning twice the distance in the east-west direction.

the FDTD algorithm. Fig. 4 illustrates a means to mitigate this problem by merging pairs of adjacent cells of the TM plane in the west-east direction, effectively halving the cell eccen- tricity. This process can be repeated several times as the grid approaches a pole, allowing the user to specify a maximum allowable cell eccentricity. 
The required algorithm is now presented. Again applying Ampere's law in integral form, the FDTD time-stepping relation for the $E_{z}$ field at the center of the merged cell is given by

$$
\begin{aligned}
& E_{z}^{n+1}(i, j, k) \\
& =E_{z}^{n}(i, j, k)+\frac{\Delta t}{\varepsilon_{0} S(j, k)} \\
& \quad \times\left\{\frac{H_{x}^{n+0.5}(i-0.5, j-0.5, k) \Delta_{\mathrm{w}-\mathrm{e}}(j-0.5, k)}{2}\right. \\
& \quad+\frac{H_{x}^{n+0.5}(i+0.5, j-0.5, k) \Delta_{\mathrm{w}-\mathrm{e}}(j-0.5, k)}{2} \\
& \quad-H_{x}^{n+0.5}(i, j+0.5, k) \Delta_{\mathrm{w}-\mathrm{e}}(j+0.5, k) \\
& \quad+\left[H_{y}^{n+0.5}(i+1, j, k)\right. \\
& \left.\left.\quad-H_{y}^{n+0.5}(i-1, j, k)\right] \Delta_{\mathrm{s}-\mathrm{n}}\right\} .
\end{aligned}
$$

The following $E_{z}$ fields within the merged cell, required for the subsequent magnetic-field updates, are obtained by linearly interpolating the $E_{z}$ field quantities calculated in (9):

$$
\begin{aligned}
E_{z}^{n+1} & (i-0.5, j, k) \\
= & \frac{\left[3 E_{z}^{n+1}(i, j, k)+E_{z}^{n+1}(i-2, j, k)\right]}{4} \\
E_{z}^{n+1}(i+0.5, j, k) & \\
\quad= & \frac{\left[3 E_{z}^{n+1}(i, j, k)+E_{z}^{n+1}(i+2, j, k)\right]}{4} .
\end{aligned}
$$

Now, the time-stepping algorithm for the merged cell can be completed by specifying the updates for the $H_{x}$ and $H_{y}$ fields at the periphery of the cell, for example

$$
\begin{aligned}
& H_{x}^{n+1.5}(i-0.5, j-0.5, k) \\
&= H_{x}^{n+0.5}(i-0.5, j-0.5, k) \\
&+\frac{\Delta t}{\mu_{0} \Delta_{\mathrm{r}}}\left[E_{y}^{n+1}(i-0.5, j-0.5, k+0.5)\right. \\
&\left.-E_{y}^{n+1}(i-0.5, j-0.5, k-0.5)\right] \\
&+\frac{\Delta t}{\mu_{0} \Delta_{\mathrm{s}-\mathrm{n}}}\left[E_{z}^{n+1}(i-0.5, j-1, k)\right. \\
&\left.-E_{z}^{n+1}(i-0.5, j, k)\right] \\
& H_{x}^{n+1.5}(i+0.5, j-0.5, k) H_{x}^{n+0.5}(i+0.5, j-0.5, k) \\
&+\frac{\Delta t}{\mu_{0} \Delta_{\mathrm{r}}}\left[E_{y}^{n+1}(i+0.5, j-0.5, k+0.5)\right. \\
&\left.-E_{y}^{n+1}(i+0.5, j-0.5, k-0.5)\right] \\
&+\frac{\Delta t}{\mu_{0} \Delta_{\mathrm{s}-\mathrm{n}}}\left[E_{z}^{n+1}(i+0.5, j-1, k)\right. \\
& H_{y}^{n+1.5}\left(i+E_{z}^{n+1}(i+0.5, j, k)\right] \\
&= H_{y}^{n+0.5}(i+1, k) \\
&+\frac{\Delta t}{\mu_{0} \Delta_{\mathrm{r}}}\left[E_{x}^{n+1}(i+1, j, k-0.5)\right. \\
&-\frac{\Delta t}{\mu_{0} \Delta_{\mathrm{w}-\mathrm{e}}(j, k)}\left[E_{z}^{n+1}(i+2, j, k)\right. \\
&\left.-E_{z}^{n+1}(i, j, k)\right]
\end{aligned}
$$

$$
\begin{aligned}
H_{x}^{n+1.5}(i, j+0.5, k) & \\
= & H_{x}^{n+0.5}(i, j+0.5, k) \\
& +\frac{\Delta t}{\mu_{0} \Delta_{\mathrm{r}}}\left[E_{y}^{n+1}(i, j+0.5, k+0.5)\right. \\
& \left.\quad-E_{y}^{n+1}(i, j+0.5, k-0.5)\right] \\
& +\frac{\Delta t}{\mu_{0} \Delta_{\mathrm{s}-\mathrm{n}}}\left[E_{z}^{n+1}(i, j, k)-E_{z}^{n+1}(i, j+1, k)\right] .
\end{aligned}
$$

\section{West-East Grid Wraparound (Periodic Boundary Condition)}

The grid wraparound (periodic boundary condition) in the west-east direction completes the FDTD algorithm for the TM components. For each row $j$ of grid column $i=1$ in Fig. 1, this is implemented by the following special time-stepping relation for $H_{y}$ at the west boundary of the grid

$$
\begin{aligned}
& H_{y}^{n+1.5}(0.5, j, k)= H_{y}^{n+0.5}(0.5, j, k) \\
&+ \frac{\Delta t}{\mu_{0} \Delta_{\mathrm{r}}}\left[E_{y}^{n+1}(0.5, j, k-0.5)\right. \\
&\left.-E_{y}^{n+1}(0.5, j, k+0.5)\right] \\
&+\frac{\Delta t}{\mu_{0} \Delta_{\mathrm{w}-\mathrm{e}}(j, k)}\left[E_{z}^{n+1}(1, j, k)\right. \\
&\left.\quad-E_{z}^{n+1}(2 M, j, k)\right] .
\end{aligned}
$$

Upon obtaining each such $H_{y}$ value, the grid wraparound is completed by implementing the following special relation for each row $j$ of grid column $i=2 M$ for $H_{y}$ at the east boundary of the grid:

$$
H_{y}^{n+1.5}(2 M+0.5, j, k)=H_{y}^{n+0.5}(0.5, j, k) .
$$

Steps (15a) and (15b) apply to all grid rows, whether containing trapezoidal or triangular cells.

\section{THE FDTD ALgORITHM, $\mathrm{TE}_{\mathrm{z}}$ COMPONENTS}

\section{A. Basic Algorithm}

Similarly, Faraday's law in integral form [14] can be applied to develop an FDTD time-stepping relation for the magnetic field $H_{z}$ at the center of the $(i+0.5, j+0.5, k+0.5)$ th trapezoidal grid cell. For example, referring to Fig. 3(a), we have

$$
\begin{aligned}
& H_{z}^{n+1.5}(i+0.5, j+0.5, k+0.5) \\
& =H_{z}^{n+0.5}(i+0.5, j+0.5, k+0.5) \\
& \quad+\frac{\Delta t}{\mu_{0} S(j+0.5, k+0.5)} \\
& \times\left\{E_{x}^{n}(i+0.5, j+1, k+0.5) \Delta_{\mathrm{w}-\mathrm{e}}(j+1, k+0.5)\right. \\
& \quad-E_{x}^{n}(i+0.5, j, k+0.5) \Delta_{\mathrm{w}-\mathrm{e}}(j, k+0.5) \\
& \quad+\left[E_{y}^{n}(i, j+0.5, k+0.5)\right. \\
& \left.\left.\quad-E_{y}^{n}(i+1, j+0.5, k+0.5)\right] \Delta_{\mathrm{s}-\mathrm{n}}\right\} .
\end{aligned}
$$




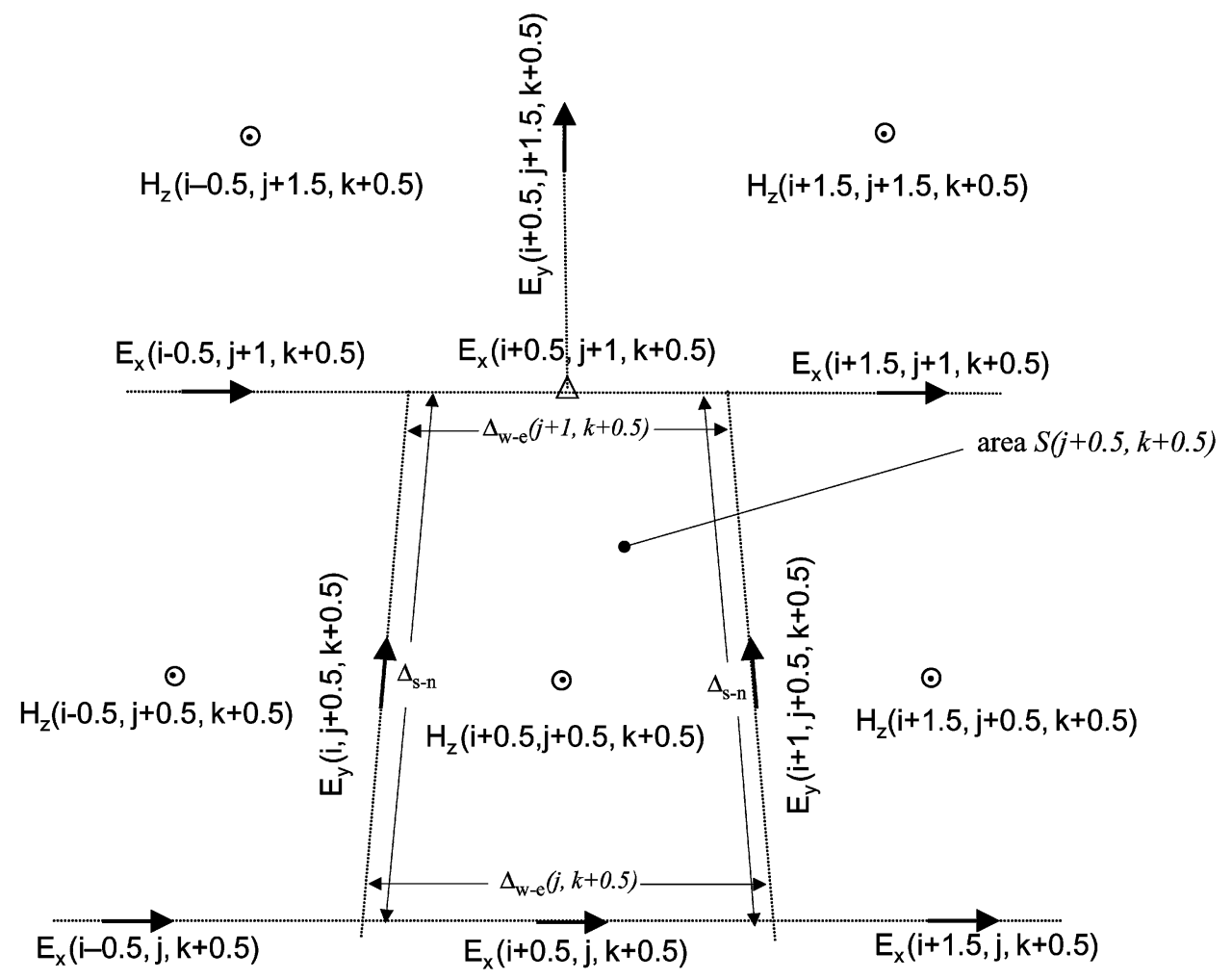

Fig. 5. Details of the $\mathrm{TE}_{\mathrm{z}}$-plane grid-cell geometry in the northern hemisphere at the transition between three adjacent regular cells and two cells each spanning twice the distance in the east-west direction.

Similarly, referring to Fig. 3(b), the update for $H_{z}$ directly at the north pole $(i=0.5, j=M+0.5)$ is given by

$$
\begin{aligned}
H_{z}^{n+1.5} & (0.5, M+0.5, k+0.5) \\
= & H_{z}^{n+0.5}(0.5, M+0.5, k+0.5) \\
& -\frac{\Delta t}{\mu_{0} S(M+0.5, k+0.5)} \\
& \times\left\{\sum_{X=1}^{N} E_{x}^{n}(X-0.5, M, k+0.5)\right. \\
& \left.\times \Delta_{\mathrm{w}-\mathrm{e}}(M, k+0.5)\right\}
\end{aligned}
$$

where $\Delta_{\mathrm{w}-\mathrm{e}}(M, k+0.5)$ is given by (2b) for the case $j=M, N$ is the number of $E_{x}$ components surrounding the $H_{z}$ component directly at the pole, and

$$
S(M+0.5, k+0.5)=\frac{N \Delta_{\mathrm{w}-\mathrm{e}}(M, k+0.5)^{2}}{4 \tan \left(\frac{\pi}{N}\right)} .
$$

Expressions analogous to (17) and (18) can be derived for $H_{z}$ directly at the south pole $(i=0.5, j=0.5)$.

The basic FDTD time-stepping algorithm is completed by specifying the updates for the $E_{x}$ and $E_{y}$ fields. For example, referring to the trapezoidal grid cell shown in Fig. 3(a), we have

$$
\begin{gathered}
E_{x}^{n+1}(i+0.5, j, k+0.5)=E_{x}^{n}(i+0.5, j, k+0.5) \\
+\frac{\Delta t}{\varepsilon_{0} \Delta_{r}}\left[H_{y}^{n+0.5}(i+0.5, j, k)\right. \\
\left.-H_{y}^{n+0.5}(i+0.5, j, k+1)\right] \\
+\frac{\Delta t}{\varepsilon_{0} \Delta_{\mathrm{s}-\mathrm{n}}}\left[H_{z}^{n+0.5}(i+0.5, j+0.5, k+0.5)\right. \\
\left.-H_{z}^{n+0.5}(i+0.5, j-0.5, k+0.5)\right](19)
\end{gathered}
$$

$$
\begin{gathered}
E_{y}^{n+1}(i, j+0.5, k+0.5)=E_{y}^{n}(i, j+0.5, k+0.5) \\
+\frac{\Delta t}{\varepsilon_{0} \Delta_{r}}\left[H_{x}^{n+0.5}(i, j+0.5, k+1)\right. \\
\left.-H_{x}^{n+0.5}(i, j+0.5, k)\right] \\
+\frac{\Delta t}{\varepsilon_{0} \Delta_{\mathrm{w}-\mathrm{e}}(j+0.5, k+0.5)} \\
\times\left[H_{z}^{n+0.5}(i-0.5, j+0.5, k+0.5)\right. \\
\left.\quad-H_{z}^{n+0.5}(i+0.5, j+0.5, k+0.5)\right] .
\end{gathered}
$$

\section{B. Merging Trapezoidal Grid Cells Approaching the Polar Regions}

As stated earlier, near the north and south poles the geometrical eccentricity of each trapezoidal cell, $\Delta_{\mathrm{s}-\mathrm{n}} / \Delta_{\mathrm{w}-\mathrm{e}}=$ $\Delta \theta /(\Delta \phi \sin \theta)$, can become quite large for a constant value of $\Delta \phi$, thereby degrading the numerical stability and efficiency of the FDTD algorithm. Fig. 5 illustrates a means to mitigate this problem by merging pairs of adjacent cells in the TE plane in the west-east direction, effectively halving the cell eccentricity. This process can be repeated several times as the grid approaches a pole, allowing the user to specify a maximum allowable cell eccentricity.

The required algorithm is now presented. The following $E_{x}$ field at the boundary of the merged cell, required for the subsequent magnetic-field update, is obtained by linearly interpolating the $E_{x}$ field quantities:

$$
\begin{aligned}
& E_{x}^{n+1}(i+0.5, j+1, k+0.5) \\
& =\frac{\left[E_{x}^{n}(i-0.5, j+1, k+0.5)+E_{x}^{n}(i+1.5, j+1, k+0.5)\right]}{2} .
\end{aligned}
$$


Applying Faraday's law in integral form, the FDTD time-stepping relation for the $H_{z}$ field below the center of the merged cell is then given by

$$
\begin{aligned}
H_{z}^{n+1.5}(i+0.5, j+0.5, k+0.5) & \\
= & H_{z}^{n+0.5}(i+0.5, j+0.5, k+0.5) \\
& +\frac{\Delta t}{\mu_{0} S(j+0.5, k+0.5)} \\
\times & \left\{E_{x}^{n}(i+0.5, j+1, k+0.5) \Delta_{\mathrm{w}-\mathrm{e}}(j+1, k+0.5)\right. \\
& \quad-E_{x}^{n}(i+0.5, j, k+0.5) \Delta_{\mathrm{w}-\mathrm{e}}(j, k+0.5) \\
& +\left[E_{y}^{n}(i, j+0.5, k+0.5)\right. \\
& \left.\left.\quad-E_{y}^{n}(i+1, j+0.5, k+0.5)\right] \Delta_{\mathrm{s}-\mathrm{n}}\right\} \cdot \quad \text { (22) }
\end{aligned}
$$

The time-stepping algorithm for the merged cell can be completed by specifying the updates for the $E_{x}$ and $E_{y}$ fields at the periphery of the cell. For example, the following algorithm is used to update the $E_{x}$ components directly above each $H_{z}(I+$ $0.5, j+0.5, k+0.5)$, for any even integer $I$, where $0 \leq I \leq 2 M$ :

$$
\begin{array}{r}
E_{x}^{n+1}(i+0.5, j, k+0.5)=E_{x}^{n}(i+0.5, j, k+0.5) \\
+\frac{\Delta t}{\varepsilon_{0} \Delta_{r}}\left[H_{y}^{n+0.5}(i+0.5, j, k)\right. \\
\left.-H_{y}^{n+0.5}(i+0.5, j, k+1)\right] \\
+\frac{\Delta t}{\varepsilon_{0} \Delta_{\mathrm{s}-\mathrm{n}}}\left[H_{z}^{n+0.5}(i+0.5, j+0.5, k+0.5)\right. \\
\left.-H_{z}^{n+0.5}(i+0.5, j-0.5, k+0.5)\right] .
\end{array}
$$

The $E_{x}$ field below each $H_{z}$ is updated as follows:

$$
\begin{gathered}
E_{x}^{n+1}(i+0.5, j, k+0.5)=E_{x}^{n}(i+0.5, j, k+0.5) \\
+\frac{\Delta t}{\varepsilon_{0} \Delta_{r}}\left[H_{y}^{n+0.5}(i+0.5, j, k)\right. \\
\left.-H_{y}^{n+0.5}(i+0.5, j, k+1)\right] \\
+\frac{\Delta t}{\varepsilon_{0} \Delta_{\mathrm{s}-\mathrm{n}}}\left[H_{z}^{n+0.5}(i+0.5, j+0.5, k+0.5)\right. \\
\left.\quad-H_{z}^{n+0.5}(i+0.5, j-0.5, k+0.5)\right] .
\end{gathered}
$$

Each $E_{y}$ field at the periphery of the cell can be updated as follows:

$$
\begin{gathered}
E_{y}^{n+1}(i, j+0.5, k+0.5)=E_{y}^{n}(i, j+0.5, k+0.5) \\
+\frac{\Delta t}{\varepsilon_{0} \Delta_{r}}\left[H_{x}^{n+0.5}(i, j+0.5, k+1)\right. \\
\left.-H_{x}^{n+0.5}(i, j+0.5, k)\right] \\
+\frac{\Delta t}{\varepsilon_{0} \Delta_{\mathrm{w}-\mathrm{e}}(j+0.5, k+0.5)} \\
\times\left[H_{z}^{n+0.5}(i-0.5, j+0.5, k+0.5)\right. \\
\left.\quad-H_{z}^{n+0.5}(i+0.5, j+0.5, k+0.5)\right] .
\end{gathered}
$$

\section{West-East Grid Wraparound (Periodic Boundary Condition)}

The grid wraparound (periodic boundary condition) in the west-east direction completes the FDTD algorithm for the TE

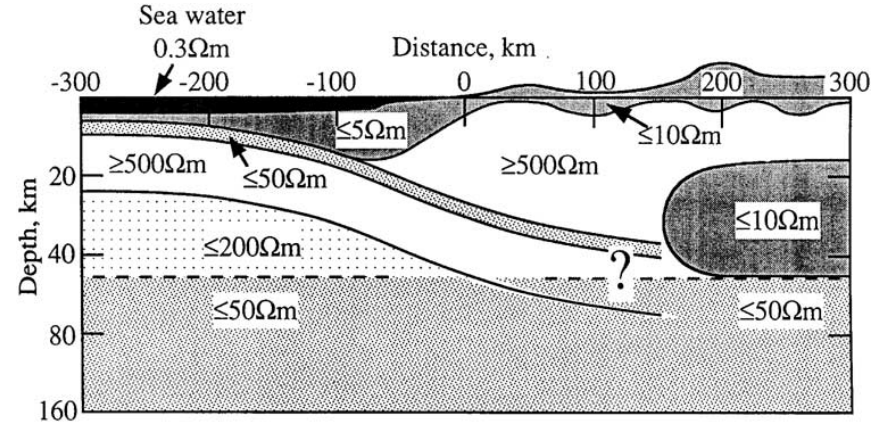

Fig. 6. Conductivity values used for the lithosphere according to whether the space lattice point is located directly below an ocean or within a continent [23].

components. For each row $j$ of grid column $i=0.5$, this is implemented by the following special time-stepping relation for $H_{z}$ at the west boundary of the grid:

$$
\begin{aligned}
& H_{z}^{n+1.5}(0.5, j+0.5, k+0.5)=H_{z}^{n+0.5}(0.5, j+0.5, k+0.5) \\
& +\frac{\Delta t}{\mu_{0} S(j+0.5, k+0.5)} \\
& \times\left\{E_{x}^{n}(0.5, j+1, k+0.5) \Delta_{\mathrm{w}-\mathrm{e}}(j+1, k+0.5)\right. \\
& \quad-E_{x}^{n}(0.5, j, k+0.5) \Delta_{\mathrm{w}-\mathrm{e}}(j, k+0.5) \\
& \quad+\left[E_{y}^{n}(2 M, j+0.5, k+0.5)\right. \\
& \left.\left.\quad \quad-E_{y}^{n}(1, j+0.5, k+0.5)\right] \Delta_{\mathrm{s}-\mathrm{n}}\right\}
\end{aligned}
$$

Upon obtaining each such $H_{z}$ value, the grid wraparound is completed by implementing the following special relation for each row $j$ of grid column $i=2 M+0.5$ for $H_{z}$ at the east boundary of the grid:

$H_{z}^{n+1.5}(2 M+0.5, j+0.5, k+0.5)=H_{z}^{n+1.5}(0.5, j+0.5, k+0.5)$.

\section{VALIDATION STUDY}

In this paper, our goal is to validate our new 3-D FDTD model by comparing its predicted ELF propagation characteristics with the data reported in [21]. Our model utilizes topographic and bathymetric data from the NOAA-NGDC "Global Relief CD-ROM" [22]. These data are mapped onto our 3-D space lattice with an assumed resolution of $40 \times 40 \times 5 \mathrm{~km}$ at the equator. This cell size is a compromise choice to meet the following set of requirements.

1) Limit the space-lattice size to permit its residence in memory on our laboratory's 2-GB workstation.

2) Resolve ELF wavelengths of $800 \mathrm{~km}$ or greater (frequencies of $375 \mathrm{~Hz}$ or less) with 20 or more cells per wavelength.

3) Provide one-to-two cell resolution for the radial extent (height and depth) of the Earth's oceans and mountain ranges.

For the lithosphere, conductivity values are assigned according to Fig. 6 [23], depending upon whether the space lattice point is located directly below an ocean or within a continent. For the atmosphere, the exponential conductivity profile used in [24] is assumed. This permits the most straightforward comparison of the present FDTD modeling results with the data reported in [16] since ELF propagation is crucially affected 

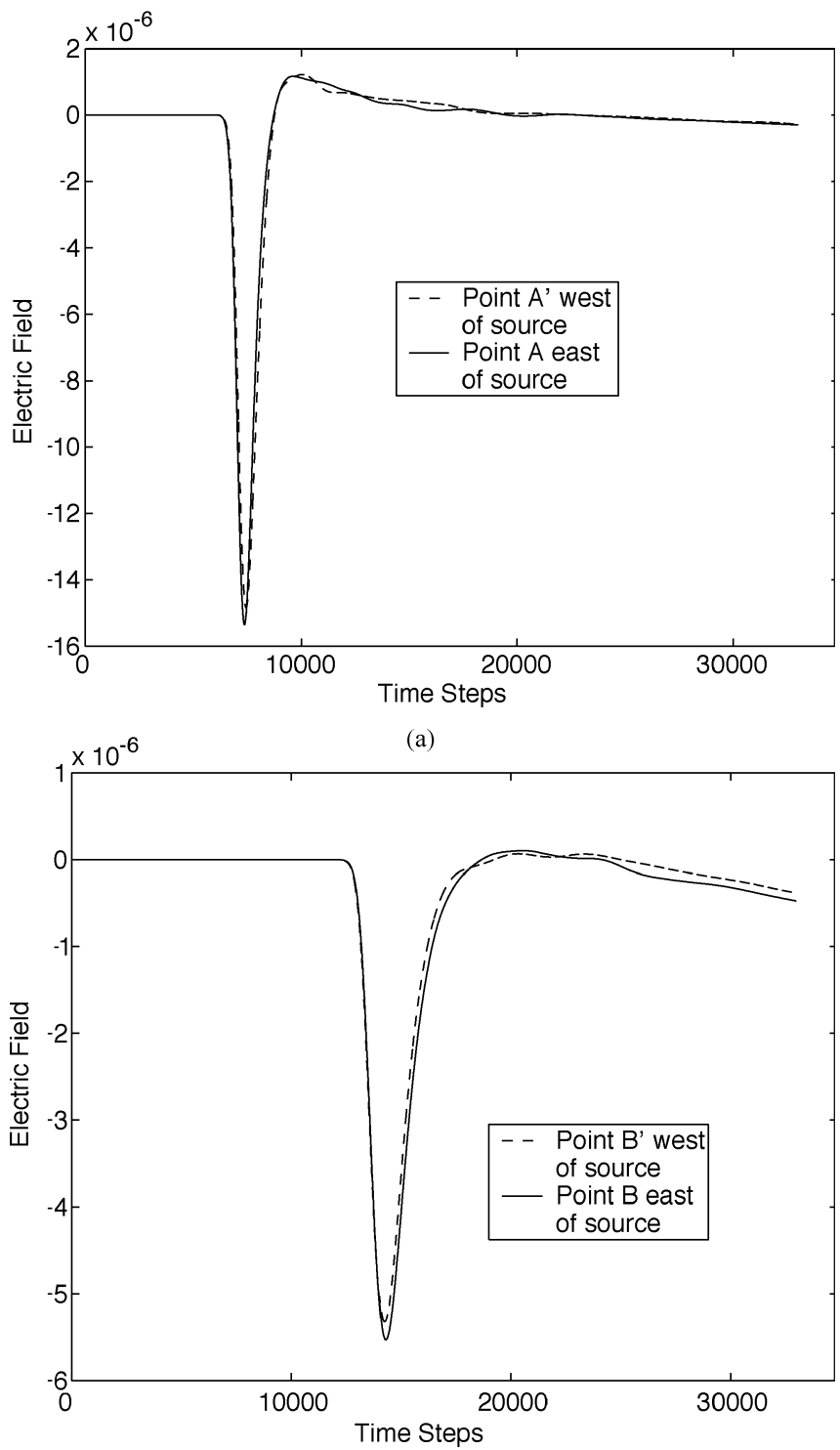

(b)

Fig. 7. FDTD-calculated temporal response observed at the equator directly east and west of the source: (a) at points $A$ and $A^{\prime}$ located $1 / 4$ of the distance to the antipode; (b) at points $B$ and $B^{\prime}$ located at $1 / 2$ of the distance to the antipode.

by the ionosphere characteristics. Note that, however, this model is capable of significantly greater ionospheric detail, i.e., day-to-night transitions, anisotropy, etc., than that possible using the analysis of [21].

We excite our model with a vertical, 5-km-long current pulse having a Gaussian time-waveform with a $1 / \mathrm{e}$ full-width of $480 \Delta t$, where $\Delta t=3.0 \mu$ s. To ensure a smooth onset of the excitation, the temporal center of this pulse is at $960 \Delta t$. This current pulse is located just above the Earth's surface on the equator at longitude $47^{\circ} \mathrm{W}$.

Fig. 7(a) graphs time-waveforms of the FDTD-calculated radial E-field at two observation points $A$ and $A^{\prime}$ on the Earth's surface at the equator directly east and west of the source at $1 / 4$ of the distance to the antipode. Fig. 7(b) graphs the FDTD-calculated time-waveforms at points $B$ and $B^{\prime}$ at the equator directly east and west of the source at $1 / 2$ of the distance to the

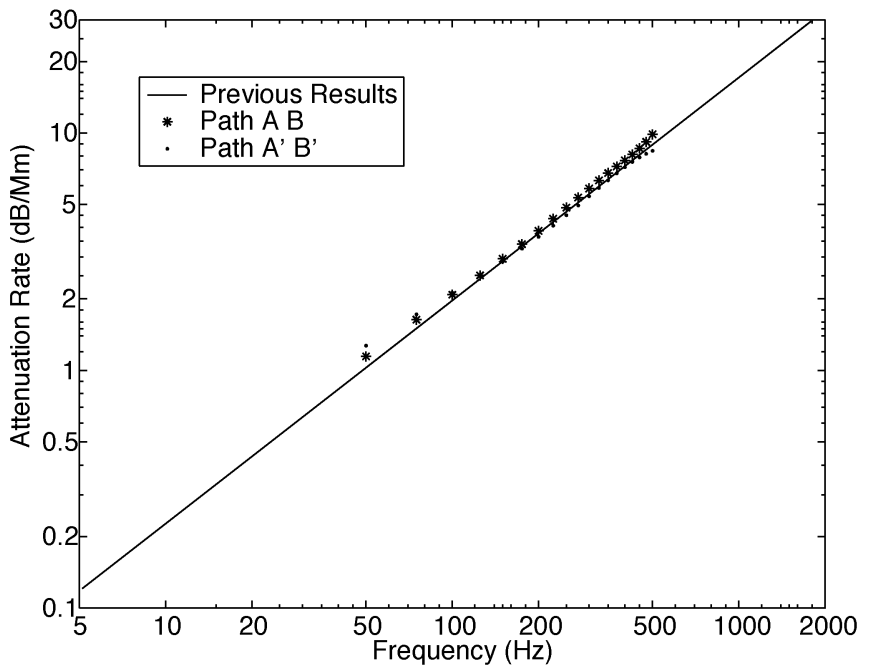

Fig. 8. Comparison between the FDTD-calculated ELF propagation attenuation versus frequency over paths $A B$ and $A^{\prime} B^{\prime}$ with the results reported in [21].

antipode. Note that waveform pair $A, A^{\prime}$ is not identical; similarly, waveform pair $B, B^{\prime}$ is not identical. The observed lack of symmetry is caused by a corresponding lack of symmetry of the lithosphere geometry in this region. Further, note the evolution of a "slow-tail" response similar to that predicted in [25].

Fig. 8 compares the FDTD-calculated ELF propagation attenuation versus frequency over paths $A B$ and $A^{\prime} B^{\prime}$ with the results reported in [21]. The FDTD data are obtained by forming the ratio of the discrete Fourier transforms (DFTs) of the time-waveforms shown in Fig. 7(a) and (b). The time-waveforms at points $A$ and $B$ are truncated at 22849 and 24165 time steps, respectively; and the time-waveforms at points $A^{\prime}$ and $B^{\prime}$ are truncated at 22737 and 25023 time steps, respectively (i.e., at each zero-crossing preceding the slow-tail response). ${ }^{2}$ Over the frequency range $50-500 \mathrm{~Hz}$, the FDTD-computed propagation attenuation values agree with the results of [21] to within about $\pm 0.5 \mathrm{~dB} / \mathrm{Mm}$ over path $A B$ and within about \pm 1.0 $\mathrm{dB} / \mathrm{Mm}$ over path $A^{\prime} B^{\prime}$.

\section{CONCLUSION}

This paper has reported the application of a computationally efficient FDTD technique to model impulsive ELF propagation within the entire Earth-ionosphere cavity. Periodic boundary conditions are used in conjunction with a spherical-coordinate, 3-D FDTD space lattice, which wraps around the complete Earth-sphere. Adaptive combination of adjacent grid cells in the east-west direction minimizes grid-cell eccentricity upon approaching the poles and hence maintains Courant stability for relatively large time steps. This technique permits direct, 3-D time-domain calculation of impulsive, round-the-world ELF

\footnotetext{
${ }^{2}$ We must window the FDTD-calculated time-waveforms in this manner because the slow-tail response persists after the appearance of the signal arriving over the equatorial long path. Therefore, the slow-tail response cannot be timestepped to its conclusion in isolation. The required windowing process in time results in a frequency window of approximately $50-500 \mathrm{~Hz}$ within which our FDTD results can be validly compared with the benchmark data of [21]. This is because errors in the DFTs occur below about $50 \mathrm{~Hz}$ due to the absence of the slow-tail response, while errors occur above about $500 \mathrm{~Hz}$ due to the truncation of the data records.
} 
propagation accounting for arbitrary horizontal as well as vertical geometrical and electrical inhomogeneities/anisotropies of the excitation, ionosphere, lithosphere, and oceans. A standard laboratory workstation (the Dell 530) is sufficient to generate high-resolution (approximately $40 \times 40 \times 5 \mathrm{~km}$ ) modeling results for all components of the Earth-ionosphere cavity within $\pm 100 \mathrm{~km}$ of sea level.

The new model is verified by comparing the FDTD calculations for ELF propagation attenuation with corresponding data reported in the literature. In our ongoing work, we are using teraflops supercomputing resources to exercise this model for both very-high-resolution and very-long-time-duration simulations, aiming at an improved understanding of the coupling between the Earth's local crustal dynamics and its global electrodynamics. In this regard, we expect that this model will eventually be coupled with emerging geophysical codes now under development for the study of seismic phenomena, including earthquake precursors. ${ }^{3}$

\section{REFERENCES}

[1] J. R. Wait, "Terrestrial propagation of very-low-frequency radio waves, a theoretical investigation," J. Res. Nat. Bur. Stand., vol. 64D, p. 153, 1960

[2] K. G. Budden, "The influence of the Earth's magnetic field on radio propagation by waveguide modes," in Proc. Royal Soc. A, vol. 265, 1962, p. 538.

[3] J. R. Wait, "Earth-ionosphere cavity resonances and the propagation of ELF radio waves," Radio Sci., vol. 69D, p. 1057, 1965.

[4] — , "Distortion of ELF pulses after propagation through an antipode," J. Geophys. Res., vol. 74, pp. 2982-2986, 1969.

[5] — Electromagnetic Waves in Stratified Media. New York: Pergamon, 1970.

[6] — - "Reflection of VLF radio waves at a junction in the earth-ionosphere waveguide," IEEE Trans. Electromagn. Compat., vol. 34, p. 4 1992.

[7] S. A. Cummer, U. S. Inan, T. F. Bell, and C. P. Barrington-Leigh, "ELF radiation produced by electrical currents in sprites," Geophys. Res. Lett., vol. 25, p. 1281, 1998.

[8] E. R. Williams, "The Schumann resonance-A global tropical thermometer," Science, vol. 256, p. 1184, 1992.

[9] D. W. Strangway, C. M. Swift, and R. C. Holmer, "The application of audio-frequency magnetotellurics to mineral exploration," Geophys. vol. 38, p. 1159, 1975.

[10] M. Parrot, "Statistical study of ELF/VLF emissions recorded by a lowaltitude satellite during seismic events," J. Geophys. Res., vol. 99, pp. 323-339, 1994

[11] J.-P. Berenger, "A FDTD scheme for the computation of VLF-LF propagation in the anisotropic earth-ionosphere waveguide," Ann. Telecommun., vol. 54, no. 5-6, pp. 297-310, 1999.

[12] — "FDTD computation of VLF-LF propagation in the Earth-ionosphere waveguide," Ann. Telecommun., vol. 57, no. 11-12, pp. $1059-1090,2002$

[13] S. A. Cummer, "Modeling electromagnetic propagation in the Earthionosphere waveguide," IEEE Trans. Antennas Propagat., vol. 48, p. 1420 , Sept. 2000.

[14] A. Taflove and S. C. Hagness, Computational Electrodynamics: The Finite-Difference Time-Domain Method, 2nd ed. Norwood, MA: Artech House, 2000.

[15] J. Simpson and A. Taflove, "Two-dimensional FDTD modeling of impulsive ELF antipodal propagation about the Earth-sphere," in Proc. IEEE AP-S Int. Symp., vol. 3, San Antonio, TX, June 2002, pp. 678-681.

[16] - "Two-dimensional FDTD model of antipodal ELF propagation and Schumann resonance of the earth," IEEE Antennas Wireless Propagat. Lett., vol. 1, pp. 53-56, 2002.

[17] — "Global three-dimensional FDTD modeling of impulsive ELF propagation about the Earth," in Proc. IEEE AP-S Int. Symp., Columbus, OH, June 2003, pp. 940-944.

${ }^{3} \mathrm{APEC}$ Cooperation for Earthquake Simulation (ACES): http://shake2.earthsciences.uq.edu.au/ACES/.
[18] M. Hayakawa and T. Otsuyama, "FDTD analysis of ELF wave propagation in inhomogeneous subionosphereic waveguide models," ACES J. vol. 17 , no. 3, pp. 239-244, Nov. 2002.

[19] T. Otsuyama, D. Sakuma, and M. Hayakawa, "FDTD analysis of ELF wave propagation and Schumann resonances for a subionospheric waveguide model," Radio Sci., to be published.

[20] R. Holland, "THREDS: A finite-difference time-domain EMP code in 3D spherical coordinates," IEEE Trans. Nucl. Sci., vol. NS-30, no. 6, pp. 4592-4595, 1983.

[21] P. Bannister, "ELF propagation update," IEEE J. Oceanic Eng., vol. OE-9, no. 3, pp. 179-188, 1984

[22] NOAA-NGDC, Global Relief CD-ROM.

[23] J. Hermance, "Electrical conductivity of the crust and mantle," in Global Earth Physics: A Handbook of Physical Constants: AGU, 1995.

[24] P. Bannister, "The determination of representative ionospheric conductivity parameters for ELF propagation in the Earth-ionosphere waveguide," Radio Sci., vol. 20, no. 4, pp. 977-984, 1985.

[25] J. R. Wait, "On the theory of the slow tail portion of atmospheric wave forms," J. Geophys. Res., vol. 65, no. 7, pp. 1939-1946, 1960

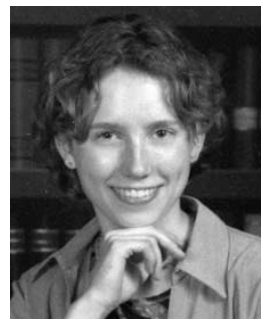

Jamesina J. Simpson (S'01) was born in Portsmouth, VA, on July 20, 1981. She is currently working toward the Ph.D. degree in electrical engineering at Northwestern University, Evanston, IL.

Since her freshman undergraduate year, she has been a Research Assistant in Prof. Taflove's Computational Electromagnetics Laboratory. Her current research interests include FDTD modeling of geophysically induced extremely low-frequency propagation about the entire Earth and FDTD modeling of novel ultrahigh-speed bandpass wireless interconnects for next-generation digital circuits.

Ms. Simpson is a Member of Tau Beta Pi. She received the Walter P. Murphy Fellowship for her graduate studies.

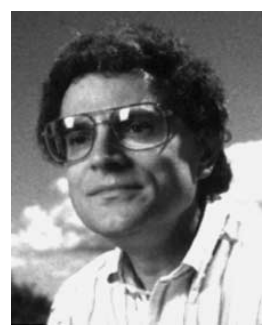

Allen Taflove (F'90) was born in Chicago, IL, on June 14, 1949. He received the B.S., M.S., and Ph.D. degrees in electrical engineering from Northwestern University, Evanston, IL, in 1971, 1972, and 1975, respectively.

After nine years as a Research Engineer with IIT Research Institute, Chicago, IL, he returned to Northwestern in 1984. Since 1988, he has been a Professor in the Department of Electrical and Computer Engineering of Northwestern's McCormick School of Engineering. Since 1972, he has pioneered basic theoretical approaches and engineering applications of finite-difference time-domain (FDTD) computational electrodynamics. He coined the FDTD acronym in a 1980 IEEE paper. Currently, FDTD is one of the most powerful and widely used methods for solving Maxwell's equations to model linear and nonlinear electromagnetic wave interactions with electrically large and complex structures. In 1995, he authored a popular textbook on this subject, Computational Electrodynamics: The Finite-Difference Time-Domain Method (Boston, MA: Artech House, June 1995). This book is now in its second edition (2000) coauthored with Prof. S. Hagness. A third edition is planned for 2005. In total, he has authored or coauthored four books, 14 invited papers or chapters in books, and approximately 250 journal papers and conference papers/abstracts. He has received 14 U.S. patents. He has been the thesis advisor of $17 \mathrm{Ph}$.D. recipients who hold professorial or technical staff positions at major institutions including research universities and national labs. Currently, he serves as the Master of Northwestern's new 140-student Slivka Residential College of Science and Engineering. He also serves as the faculty advisor to Northwestern's Undergraduate Design Competition, the student chapters of the Eta Kappa Nu and Tau Beta Pi engineering honor societies, and McCormick's Honors Program in Undergraduate Research. His research interests span much of the electromagnetic spectrum. He and his students are currently modeling electrodynamic phenomena ranging from geophysically induced extremely low-frequency wave propagation about the entire Earth to the lasing behavior of aggregates of micrometer-scale zinc-oxide particles exhibiting four-level quantum-system characteristics.

Dr. Taflove was named a C. D. McCormick Professor of Teaching Excellence in 2000 and was named to the "Highly Cited Researchers" list of the Institute for Scientific Information in 2002. 